\title{
Improving the Efficiency of Electrical Stimulation Activities After Spinal Cord Injury
}

\author{
David R. Dolbow • William R. Holcomb • \\ Ashraf S. Gorgey
}

Published online: 18 June 2014

(C) Springer Science + Business Media New York 2014

\begin{abstract}
In order to enhance spinal cord injury (SCI) rehabilitation programs using neuromuscular electrical stimulation (NMES) and functional electrical stimulation (FES) it is important to examine the manner in which muscle fibers are recruited and the dose-response relationship. A review of the literature suggests that premature force decline and early fatigue with NMES and FES activities may be alleviated with decreased current frequency and increased current intensity. Dose-response relationships with NMES and FES are dependent on the goals of interest as reversing muscle atrophy can be achieved with activities 2-3 times per week for 6 or more weeks while increasing bone mass is more limited and requires more intense activity with greater exercise frequency and duration, e.g., 3-5 days per week for at least 6-12 months. The best known protocol to elicit neurological improvement is massed practice activities-based restorative therapies (ABRT) (3-5 h per day for several weeks).
\end{abstract}

D. R. Dolbow $(\bowtie) \cdot$ W. R. Holcomb

Human Performance and Recreation Department, University of Southern Mississippi, 118 College Drive, Box 5142, Hattiesburg, MS 39406, USA

e-mail:david.dolbow@usm.edu

W. R. Holcomb

e-mail: bill.holcomb@usm.edu

\section{A. S. Gorgey}

Spinal Cord Injury Research, McGuire VA Medical Center, 1201

Broad Rock Blvd, Richmond, VA 23224, USA

e-mail: ashraf.gorgey@va.gov

\section{A. S. Gorgey}

Physical Medicine and Rehabilitation, Virginia Commonwealth University, Richmond, VA, USA
Keywords Neuromuscular electrical stimulation . Functional electrical stimulation · Spinal cord injury · Muscle fiber recruitment $\cdot$ Neuroplasticity $\cdot$ Body composition $\cdot$ Secondary health conditions

\section{Introduction}

Electrical activity is a primary component of the neurological system of all living creatures. Everything we do is linked to a series of electrochemical signals that travel throughout our bodies. When the neurological system is functioning optimally, mental and physical activities continue without interruption. However, when damage occurs within the brain or spinal cord, electrical signals can be disrupted halting communication between neurons. This can be especially devastating to the somatic motor system because it is under voluntary control and the spinal cord is the highway through which signals for functional activities are passed from the brain to skeletal muscles [1].

Alternative external methods have been used successfully to induce electrical stimulation to the body for the purpose of restoring skeletal muscle activities after disruption of natural neurotransmission due to spinal cord injury (SCI). Common terms within this practice are neuromuscular electrical stimulation (NMES) and functional electrical stimulation (FES). Although NMES and FES are often used interchangeably they can be divided into two distinct activities. NMES is the electrical stimulation of muscle to facilitate muscle action and reeducation of the muscle toward normal function [2]. FES is the utilization of multiple channel electrical stimulators controlled by a microprocessor to recruit muscles in a programmed synergistic sequence that will allow accomplishment of a specific functional movement pattern [2]. Both NMES and 
FES have proven to provide overlapping benefits toward the rehabilitation of those with SCI, however, each activity also has its' own particular strengths when it comes to restoration of function. In order to optimize electrical stimulation programs for individuals with SCI, we must look at the manner in which muscle contractions are induced as well as the relationship between "the doses of exercise" and desired physiological responses of the exerciser.

\section{Muscle Recruitment and Torque Decline with NMES/ FES}

Because most benefits of exercise are dose related, it is important to be able to sustain adequate periods of exercise in order to realize benefits. A limitation of training with FES and NMES is the inability to maintain sufficient contraction force over the training period. When training muscles with NMES using multiple repetitions, significant force decline occurs and could result in an insufficient training stimulus. During FES-assisted ambulation, participants with SCI often develop early onset fatigue which limits the activity duration [3]. When using NMES resistance training, FES-assisted ambulation, FES cycling or other electrical stimulation functional activities with SCI individuals, sufficient contraction force must be maintained to enable the activity for a prescribed training duration. It has been commonly accepted that the greater decline in force during NMES and FES activities in contrast to voluntary exercise is the result of greater fatigue. There has been a long held belief that the order of motor unit recruitment with NMES/FES is the reverse of the physiological recruitment order first described by Henneman et al. [4] as the size principle. With voluntary exercise the smaller motor units that are slow, weak but fatigue resistant are recruited first. As greater force is required the larger motor units that are fast, strong yet fatigue rapidly are then recruited. Some authors have concluded that the recruitment order is reversed with NMES/FES because the axons of the larger motor units have less resistance to electrical current [5]. Therefore, selective recruitment of the large, fast motor units would result in a more rapid fatigue than with voluntary exercise. Although this has been readily accepted as a logical explanation for the greater decline in force that has been observed with NMES and early fatigue with FES, other plausible explanations are gaining support.

Gregory and Bickel reviewed the literature and made a compelling argument that electrical stimulation does not result in a reversal of the recruitment order that occurs with voluntary exercise. In their perspective, the authors concluded that the reversal may occur with direct stimulation of the motor nerve but not with stimulation through surface electrodes that are used clinically [6]. With stimulation through surface electrodes it is likely that the recruitment is random and representative of the types of motor units in the proximity of the electrodes. Therefore, the percentage recruitment of fast motor units would depend on the percentage of fast motor units in the muscle being stimulated and the location of axonal branches relative to the placement of the surface electrodes. This recruitment pattern would likely result in slightly greater fatigue than with voluntary contractions where recruitment follows the size principle. In addition, with voluntary contractions there is the ability to delay fatigue with asynchronous recruitment which is not possible with NMES or FES. So, it is apparent that a portion of the greater force decline that occurs with electrical stimulation exercise versus voluntary exercise is the result of greater fatigue.

Logically, if the decline in force was due entirely to fatigue, longer rest intervals would negate the decline. However, Holcomb et al. reported force declines of $8.2 \%$ over 5 NMES repetitions even when rest intervals were lengthened to $2 \mathrm{~min}$ between each repetition. Under the same conditions force declines with voluntary contractions were only $2.8 \%$ [7]. Gorgey et al. [8] reported that decreasing the frequency from 100 to $25 \mathrm{~Hz}$ decreased fatigue from 76 to $39 \%$ during NMES knee extension exercise accounting for just under half of the fatigue presented. Therefore, it is offered that there is an additional contribution to the greater force decline in electrically induced activities. This additional factor is accommodation to the electrical current intensity. Randolph et al. [9] reported a significant decline in force from repetition 1 to repetition 10 while at the same time reported a significant decrease in ratings of discomfort. These results appear to suggest that accommodation to current intensity occurs and may contribute to the decline in force. Holcomb et al. provided further support by increasing NMES intensity by $5 \%$ after every other repetition in a ten repetition set. With this strategy, the force during repetition 10 was not significantly different than repetition 1 [10]. In sensory intact individuals one might expect that discomfort would increase with the increased stimulation intensity but this was not the case. There was no statistically significant difference in perceived discomfort from repetition 1 to 10 which suggests that the effects of accommodation were negated. The fact that the effects of accommodation were negated as force was maintained lends further support to the idea that accommodation contributes to fatigue in providing decline in electrically stimulated force when compared to voluntary exercise. Therefore, to counteract the decline in force and/or increase in fatigue that appears to occur over time with electrically stimulated exercise, lower frequency combined with increases in current intensity is recommended. 
One other factor to consider during NMES and FES activities is the amount of subcutaneous fat in the area where surface electrodes are placed. A recent study demonstrated that individuals with $50 \%$ greater cross-sectional area (CSA) of thigh subcutaneous fat compared to a control group required 48-59\% greater current amplitude to complete full knee extension [11].

\section{Paradigm Shift in Electrical Stimulation Neurorehabilitation}

Electrical stimulation devices were originally developed to assist with activities such as respiration, bowel and bladder function, grasping, standing, and walking activities [12]. The initial purpose was to help compensate for the loss of function due to a damaged neurological system. The prevailing belief throughout most of the twentieth century was that the central nervous system (brain and spinal cord) was in effect hard wired and once damaged restoration of function was not possible, thus the need for external compensation [13]. However, the successful restoration of significant neurological function of individuals with chronic SCI over the past couple decades has precipitated a paradigm shift in rehabilitation focus [13-15].

In addition to function restoration through FES-driven orthotics, electrical stimulation activities for individuals with SCI have two other separate areas of focus (1) enhanced neurologic function via restoration of the damaged spinal cord and (2) prevention of secondary risk factors associated with inactivity due to muscle paralysis. Concerning restoration of the spinal cord, repetitive electrical stimulation activities have been shown to promote the generation of tripotential progenitor cells, stimulate remyelination of damaged neuronal axons, and promote sprouting of new collateral axons that have potential to assist in the re-establishment of neuronal function [3, 14, $16 \bullet, 17 \bullet \cdot$. Although restoration of damaged spinal pathways in humans has not matched that in animals, positive results provide encouragement of greater potential benefits.

Along with neurological damage within the spinal cord, paralysis below the level of injury causes negative body composition changes that increase the risk of secondary conditions such as obesity, metabolic syndrome, diabetes mellitus, cardiovascular disease, and osteoporosis [18-25]. FES and NMES activities have been shown to help decrease the risk of inactivity-related health conditions by partially reversing loss of muscle and bone mass and decreasing the accumulation of fat mass that results from paralysis and inactivity [18, 24, 26-33]. Similar to restoration of neurological function in the spinal cord, beneficial results in body composition and associated secondary disease risk are thus far limited, however, significant results in this area point to greater potential benefits.

The primary electrical stimulation activities in use related to skeletal muscle activities are NMES resistance training, FES cycling, and FES-assisted ambulation [34]. These and other developing activities provide individuals with SCI an option for increasing physical activity to healthy levels, a means for exercising paralyzed muscles and produces positive body composition changes while sending health-promoting signals into the spinal cord [35••, 36]. The study of electrical stimulation activities as one component in the effort to enhance rehabilitation of individuals with SCI remains substantial as researchers continue to work toward the establishment of procedures and practices that are more efficient.

\section{NMES/FES Dose-Response}

An important area of interest concerning the optimization of FES and NMES activities is the dose-response relationship. While it can be stated that physical activity volume and all-cause mortality have an inverse linear doseresponse relationship, the components of an exercise program (intensity, frequency, and duration) vary depending on the desired benefits, i.e., increased muscle mass, decreased fat mass, increased bone mass, cardiovascular conditioning, and enhanced neuroplasticity [25, 30, 37, 38].

Activity-dependent neuroplasticity has been studied in animals and humans with varying levels of success [14, 39-41]. Described as the interface between physical and neural activity, activity-dependent neuroplasticity is the resultant increase in neurotrophic proteins that promote neuron survival and development [39]. FES cycling and FES-assisted ambulation are activities that provide potential for neuroplasticity and restoration of functional spinal pathways [3, 14, 41, 42]. The predominant theory of activity-driven SCI rehabilitation programs, e.g., activitybased restorative therapies (ABRT), promotes the need for high volume physical activity to provide positive neuronal changes. In these programs, massed practice $(3-5 \mathrm{~h}$ per day) is utilized through a combination of physical activities which include but are not limited to FES cycling, manual or electrical stimulated standing and body weight supported treadmill training [13-15].

Task specific training has been reported to be optimal for restoration of functional activities after SCI, especially concerning neuro-adaptive central pattern generation [43]. However, FES-assisted ambulation with paralytic lower extremities has double the energy cost of able-bodied walking and is much slower [3], thus unable to singularly produce the characteristics of massed practice which promote positive neurological changes. For this reason, 
multiple activities are required during an ABRT program. FES cycling is an integral ABRT component because it is typically performed for 30-60 min per session and at a cycling speed of 50 revolutions per minute (rpm) provides adequate massed practice stimulation within the spinal cord $[3,15,39,44]$. Sixty minutes of FES cycling can provide spinal impulses equivalent to performing 6,000 steps. Along with high exercise frequency and duration, the electrical intensity of FES activity must be great enough to safely perform the activity throughout each session. When sensation is present, comfort level must take precedence when determining the electrical intensity of the activity because it can be potentially painful.

ABRT program exercise frequency is typically 5 days per week for a duration of 2-6 weeks. Sessions are massed in part to elicit as much neuro-recovery as possible while the participants have access to a myriad of neurorehabilitation equipment (i.e., FES cycles, body weight supported treadmills, therapeutic pools) and access to SCI rehabilitation specialists as community fitness and wellness centers customarily provide access to neither.

Exercise parameters to enhance body composition depend on the component of interest. Increasing muscle mass of a specific muscle group after SCI may be most optimally achieved through resistive training via NMES. Mahoney et al. [45] and Gorgey and Shepherd [46] found increases in the CSA of quadriceps muscles by 35-39\% after NMES resistance training (4 sets of 10 repetitions with 2 min rest intervals, 2 times per week for 12 weeks). Gorgey et al. [47] also found that inducing muscle hypertrophy in the quadriceps muscles via NMES also co-activates the surrounding muscles causing increased CSA in the hamstrings and hip adductors.

Less efficient for individual muscle group hypertrophy but with the capacity to affect a greater number of muscle groups in a single exercise session, FES-lower extremities cycling (LEC) has been shown to improve total body and legs muscle mass from 3.3 to $12 \%$ with $2-3$ sessions weekly over 9 weeks up to 1 year [27, 44, 48, 49]. Likewise, FES-LEC 2-3 times per week for 10 weeks increased the CSA of leg muscles by $10 \%[50,51]$. Frotzler et al. found a $35 \%$ increase in thigh muscle CSA and a $16 \%$ decrease in thigh fat CSA after 12 months of FES-LEC. Participants cycled for an average of 58 min 3.7 times per week [26]. Fornusek et al. demonstrated that slower speeds were superior to faster speeds as they found that mid-thigh girth increased by $6.6 \%$ after 6 weeks of 3 times per week FES-LEC at speeds of $10 \mathrm{rpm}$ as compared to a $3.6 \%$ increase with a speed of $50 \mathrm{rpm}$ [52]. A primary factor being that the slower cadence of cycling allowed greater resistance levels and increased stress on muscle fibers.

The metabolism rate of bone is much slower than for muscle, thus changes in bone mineral density (BMD) require more time. Whereas changes in muscle mass due to electrical stimulation may be seen in weeks, changes in BMD are likely not seen until after 6 months. In a review of literature on the effects of aging and electrical stimulation on bone mass after SCI, Dolbow et al. found no studies using an FES-LEC frequency of $\leq 2$ times per week reporting improvements in BMD. To the contrary, FES-LEC frequencies of 3 times per week or more showed mixed results with 5 times per week producing the best results [30].

Chen et al. [53] found an 11 and $13 \%$ increase in BMD at the distal femur and proximal tibia after FES-LEC 5 days per week for 6 months. Frotzler et al. demonstrated that trabecular and total BMD in the distal femur increased by 14 and $7 \%$, respectively, after participants with SCI performed FES-LEC an average 58 min per session, 3.7 times per week for 12 months. During this study, each participant cycled at their highest possible workload [26].

Shields and Dudley-Javoroski used NMES on the soleus muscle 5 times per week for $2-3$ years at an intensity 1.5 times their body weight and found a $31 \%$ increase in trabecular BMD at the distal femur [54]. In a follow-up study, the authors reduced the NMES load to 1.1 times body weight 5 times per week for 6-11 months and found no change in BMD indicating the importance of intensity of exercise for BMD improvements [55].

As in muscle mass, NMES training may evoke greater BMD changes but is more limited in that it typically is used on one muscle group at a time whereas FES activities exercise multiple muscle groups simultaneously. This allows FES activities to apply bone enhancing stress to a greater area of bone.

Altering BMD with electrical stimulation after SCI is more difficult than creating positive change in soft tissue body components (i.e., muscle and fat). Intensity of the workload must be high; the frequency $\geq 3$ times per week and the duration of the exercise program must extend for several months in order to find change in the trabecular rich areas of bone (i.e., distal femur and proximal and distal tibia).

Individuals with SCI have an increased risk of cardiovascular disease due to muscle paralysis and decreased physical activity. The prevalence of cardiovascular disease is reported to be 3-5 times greater among individuals with SCI compared to able-bodied individuals [56-59]. Due to lack of sympathetic input and autonomic control, the cardiovascular system for individuals with injuries T6 and above has impaired responses to cardiovascular conditioning activities [36]. Faghri et al. [28] studied the effects of FES-LEC 3 times per week for 12 weeks on SCI individuals and found increased cardiac stroke volume and cardiac output. The authors concluded that these results suggest individuals with SCI are able to improve peripheral muscular and central cardiovascular fitness with FES-LEC training. Hooker et al. demonstrated an increase in peak 
$\mathrm{VO}_{2}$ with FES-LEC after participants with SCI performed FES-LEC for 30 min 2 times per week for 19 weeks [60]. Hamzaid and Davis [36] reviewed the health and fitness benefits of FES-LEC in persons with SCI and determined that although study reports varied and in some cases were contradictory, overall the observation of increased stroke volume, cardiac output, and peak $\mathrm{VO}_{2}$ provides credence for the ability of regular FES activities to promote cardiovascular benefits.

Sadowsky et al. were able to examine the benefits of FES-LEC in a cross-sectional retrospective review as part of a 29-month follow-up evaluation. Individuals that participated in FES-LEC increased an average $36 \%$ in quadriceps muscle mass and decreased intra/inter-muscular fat by $44 \%$ compared to the control group. FES-LEC participants also increased an average 20 points on the combined motor-sensory scale (American Spinal Cord Injury Association Impairment Scale) while the controls decreased by an average 9.6 points. Although this is a cross-sectional examination, the results provide evidence of the ability of FES activities to enhance neurologic and body composition health [35••].

Because of the varied disability among individuals with SCI and the resultant difficulty in designing an effective exercise protocol (intensity, frequency, duration), Perret et al. [61] simplified the process by investigating the feasibility of a healthy FES-LEC weekly exercise caloric expenditure. For SCI individuals that had been training with FES-LEC for 6 months, 4-8 h of cycling was required to expend the commonly recommended 1,000-2,200 kcal per week to reduce health risks [61]. Considering that $30 \mathrm{~min}$ of exercise daily is a commonly accepted dose for reducing health risk, the authors concluded that using the FES-LEC weekly caloric expenditure was a feasible alternative method for rating physical activity level.

\section{Limiting Factors/Safety Precautions}

When designing an exercise program for an individual with SCI all activity components are necessarily guided by the level and completeness of injury [34]. Persons with motor complete injuries require electrical stimulation to induce muscle contractions below the level of injury while incomplete injuries may have some level of voluntary motor activity. An intact sensory system requires that electrical stimulation be modified dependent upon the comfort level of the participant.

Individuals with complete SCI at T6 level and above display a compromised cardiovascular system due to impaired autonomic control and loss of input from the sympathetic nervous system resulting in bradycardia and hypotension [62]. This cardio-dysfunction blunts maximal heart rates ( $\leq 130$ beats per minute), which limits cardiac output and blood supply to exercising muscles [34]. Individuals with injuries T10 and below are more likely to have lower motor neuron dysfunction which can result in the inability to receive external electrical stimulation rendering FES ineffective [34].

Another concern is that chronic motor complete SCI results in significant bone loss leading to neurogenic osteoporosis and increased risk of fragility fractures [30]. While it has been reported that hundreds of individuals with SCI have safely performed thousands of FES activities it is important to screen potential FES activity participants for those at high risk (severely low BMD and/or history of fragility fractures) in order to limit risk of injury [30].

\section{Conclusion and Future Trends}

To date, there are no universally accepted exercise protocols for the use of NMES and FES activities for persons with SCI. General exercise guidelines defined for ablebodied individuals are not sufficient due to the complex nature of SCI. As mentioned above, exercise protocols must vary depending on the desired result and the level of compromise to involved physiological systems. This review was not meant to be exhaustive but rather to provide information from available research indicating protocols that have been successful in research and clinical settings. As SCI research and electrical stimulation technology continue to advance, the protocols presented in this review will certainly be optimized.

A major area of need in SCI rehabilitation is long-term access to electrical stimulation devices. Currently, there is no other means by which paralyzed muscle can be stimulated to exercise in order to promote health. Once initial and extended rehabilitation processes are completed in clinical settings, individuals are left without resources as community fitness centers are not equipped to accommodate the special needs of a person with SCI. New technologies have provided adaptive FES cycles and other devices that can be used in the home; however, these items remain expensive and unrealistic for many with SCI. The problem of continuum of care beyond clinical rehabilitation is a dilemma that requires a solution. Possible solutions are the development of less-expensive FES cycles and other devices or the inclusion of these devices in community fitness and wellness centers along with individuals trained in the special needs of those with SCI.

The general consensus concerning a future cure for SCI is that it will require the combination of clinical, rehabilitative, pharmacological, and technological advancements. Electrical stimulation and physical activity will certainly be part of that equation. Similarly, prevention and limiting 
neuronal damage (apoptosis) after SCI are also a very important part of this very large task.

\section{Compliance with Ethics Guidelines}

Conflict of Interest DR Dolbow, WR Holcomb and AS Gorgey declares no conflicts of interest.

Human and Animal Rights and Informed Consent All studies by DR Dolbow, WR Holcomb, and AS Gorgey involving animal and/or human subjects were performed after approval by appropriate institutional review boards. When required, written informed consent was obtained from all participants.

\section{References}

Papers of particular interest, published recently, have been highlighted as:

-• Of major importance

1. Bear MF, Connors BW, Paradiso MA. Neuroscience: exploring the brain. 3rd ed. Philadelphia, PA: Lippincott Williams \& Wilkins; 2007.

2. Shamus E, Wilson SH. The physiologic effects of therapeutic modality: intervention on the body systems. In: Prentice WE, editor. Therapeutic modalities for physical therapists. 2nd ed. New York: McGraw-Hill; 2002.

3. Hamid S, Hayek R. Role of electrical stimulation for rehabilitation and regeneration after spinal cord injury: an overview. Eur Spine J. 2008;17(9):1256-69.

4. Henneman E, Somjen G, Carpenter DO. Functional significance of cell size in spinal motoneurons. J Neurophysiol. 1965;28:560-80.

5. Kubiak RJ, Whitman KM, Johnston RM. Changes in quadriceps femoris muscle strength using isometric exercise versus electrical stimulation. J Orthop Sports Phys Ther. 1987;8:537-41.

6. Gregory CM, Bickel CS. Recruitment patterns in human skeletal muscle during electrical stimulation. Phys Ther. 2005;85(4): 358-64.

7. Holcomb WR, Rubley MD, Miller MG, Girouard TJ. The effect of rest intervals on knee-extension torque production with neuromuscular electrical stimulation. J Sport Rehabil. 2006;15: $116-24$.

8. Gorgey AS, Black CD, Elder CP, Dudley GA. Effects of electrical stimulation parameters on fatigue in skeletal muscle. J Orthop Sports Phys Ther. 2009;39(9):684-92.

9. Randolph SM, Holcomb WR, Rubley MD, Miller MG. Assessment of torque and perceived pain during ten repetitions of neuromuscular electrical stimulation. Athletic Train Sports Health Care. 2009;1:162-8.

10. Holcomb WR, Rubley MD, Randolph SM. Increasing neuromuscular electrical stimulation amplitude to reduce the decline in knee extension torque. Athletic Train Sports Health Care. 2011;3(2):63-8.

11. Gorgey AS, Cho GM, Dolbow DR, Gater DR. Differences in current amplitude evoking leg extension in individuals with spinal cord injury. NeuroRehabilitation. 2013;33(1):161-70.

12. Gater DR, Dolbow DR, Tsui B, Gorgey AS. Functional electrical stimulation therapies after spinal cord injury. NeuroRehabilitation. 2011;28:231-48.

13. Behrman AL, Bowden MG, Nair PM. Neuroplasticity after spinal cord injury and training: an emerging paradigm shift in rehabilitation and walking recovery. Phys Ther. 2006;86(10):1406-25.
14. Sadowsky CL, McDonald JW. Activity-based restorative therapies: concepts and applications in spinal cord injury-related neurorehabilitation. Dev Disabil Res Rev. 2009;15(2):112-6.

15. Martin R, Sadowsky C, Obst K, Brooke M, McDonald J. Functional electrical stimulation in spinal cord injury: from theory to practice. Top Spinal Cord Inj Rehabil. 2012;18(1):28-33.

16. • Li Q, Brus-Ramer M, Martin JH, McDonald JW. Electrical stimulation of the medullary pyramid promotes proliferation and differentiation of oligodendrocyte progenitor cells in the corticospinal tract of the adult rat. Neurosci Lett. 2010;479(2):128-33. Researchers induced 10 days of electrical stimulation of the corticospinal tract at the medullary pyramid level in adult rats. The authors reported proliferation and differentiation of oligodendrocyte progenitor cells in the corticospinal tract.

17. • Becker D, Gary DS, Rosenzweig ES, Grill WM, McDonald JW. Functional electrical stimulation helps replenish progenitor cells in the injured spinal cord of adult rats. Exp Neurol. 2010;222(2):211-8. The authors reported that after 10 days of FES stimulation to the hind legs of spine transected rats, there was an 82-86\% increase in cell birth in the lumbar spine compared to the control group. Authors also reported that the FES group had double the proportion of newly birthed tripotential progenitor cells.

18. Gater DR Jr. Obesity after spinal cord injury. Phys Med Rehabil Clin N Am. 2007;18(2):333-51.

19. Spungen AM, Adkins RH, Stewart CA, Wang J, Pierson RN Jr, Waters RL, Bauman WA. Factors influencing body composition in persons with spinal cord injury: a cross-sectional study. J Appl Physiol. 2003;95(6):2398-407.

20. Bauman WA, Spungen AM. Metabolic changes in persons after spinal cord injury. Phys Med Rehabil Clin N Am. 2000;11(1): 109-40.

21. Bauman WA, Spungen AM. Carbohydrate and lipid metabolism in chronic spinal cord injury. J Spinal Cord Med. 2001;24(4): 266-77.

22. Phillips CA. Medical criteria for active physical therapy. Physician guidelines for patient participation in a program of functional electrical rehabilitation. Am J Phys Med. 1987;66(5):269-86.

23. Kocina P. Body composition of spinal cord injured adults. Sports Med. 1997;23(1):48-60.

24. Gorgey AS, Mather KJ, Cupp HR, Gater DR. The effects of resistance training on adiposity and metabolism after spinal cord injury. Med Sci Sports Exerc. 2012;44(1):165-74.

25. Dolbow DR, Gorgey AS, Daniels JA, Adler RR, Gater DR Jr. The effects of spinal cord injury and exercise on bone mass: a literature review. NeuroRehabilitation. 2011;29(3):261-9.

26. Frotzler A, Coupaud S, Perret C, Kakebeeke TH, Hunt KJ, Nde Donaldson N, Eser P. High-volume FES-cycling partially reverses bone loss in people with chronic spinal cord injury. Bone. 2008;43:169-76.

27. Griffin L, Decker MJ, Hwang JY, Wang B, Kitchen K, Ding Z, Ivy JL. Functional electrical stimulation cycling improves body composition, metabolic and neural factors in persons with spinal cord injury. J Electromyogr Kinesiol. 2009;19(4):614-22.

28. Faghri PD, Glaser RM, Figoni SF. Functional electrical stimulation leg cycle ergometer exercise: training effects on cardiorespiratory responses of spinal cord injured subjects at rest and during submaximal exercise. Arch Phys Med Rehabil. 1992;73(11):1085-93.

29. Demchak TJ, Linderman JK, Mysiw WJ, Jackson R, Suun J, Devor ST. Effects of functional electrical stimulation cycle ergometry training on lower limb musculature in acute SCI individuals. J Sports Sci Med. 2005;4:263-71.

30. Dolbow JD, Dolbow DR, Gorgey AS, Adler RR, Gater DR. The effects of aging and electrical stimulation exercise on bone after spinal cord injury. Aging Dis. 2013;4(3):141-53. 
31. Hooker SP, Figoni SF, Rodgers MM, et al. Physiologic effects of electrical stimulation leg cycle exercise training in spinal cord injured persons. Arch Phys Med Rehabil. 1992;73(5):470-6.

32. Nash MS, Bilsker S, Marcillo AE, Isaac SM, Botelho LA, Klose KJ, Green BA, Rountree MT, Shea JD. Reversal of adaptive left ventricular atrophy following electrically stimulated exercise training in human tetraplegics. Paraplegia. 1991;29(9):590-9.

33. Brurok B, Torhaug T, Karlsen T, Leivseth G, Helgerud J, Hoff J. Effect of lower limb functional electrical stimulation pulsed isometric contractions on arm cycling peak $\mathrm{O}_{2}$ uptake in spinal cord injury individuals. J Rehabil Med. 2013;45(3):254-9.

34. Jacobs PL, Nash MS. Exercise recommendations for individuals with spinal cord injury. Sports Med. 2004;34(11):727-51.

35. • Sadowsky CL, Hammond ER, Strohl AB, Commean PK, Eby SA, Damiano DL, Wingert JR, Bae KT, McDonald JW 3rd. Lower extremity functional electrical stimulation cycling promotes physical and functional recovery in chronic spinal cord injury. J Spinal Cord Med. 2013;36(6):623-31. The authors reported on a retrospective cross-sectional evaluation of individuals with SCI that had performed FES cycling as part of an activity based treatment program. Results indicated significantly higher combined motor-sensory scores and higher levels of quadriceps muscle with lower intra/inter muscle fat. QOL scores were also higher among those that participated in FES cycling.

36. Hamzaid NA, Davis GM. Health and fitness benefits of functional electrical stimulation evoked leg exercise for spinal cord injury individuals: a position review. Top Spinal Cord Inj Rehabil. 2009; 14(4):88-121.

37. Lee IM, Skerrett PJ. Physical activity and all-cause mortality: what is the dose-response relationship? Med Sci Sports Exerc. 2001;33(6 Suppl):S459-71; discussion S493-4.

38. Loprinzi PD, Lee H, Cardinal BJ. Dose response association between physical activity and biological, demographic, and perceptions of health variables. Obesity Facts. 2013;6(4):1-21. Retrieved 12 May 2014 from http://www.karger.com/Article/ FullText/354752.

39. Dunlop SA. Activity-dependent plasticity: implications for recovery after spinal cord injury. Trends Neurosci. 2008;31(8): $410-8$.

40. Astorino TA, Tyerman N, Wong K, Harness E. Efficacy of a new rehabilitative device for individuals with spinal cord injury. J Spinal Cord Med. 2008;31(5):586-91.

41. Phadke CP, Flynn SM, Thompson FJ, Behrman AL, Trimble MH, Kukulka CG. Comparison of single bout effects of bicycle training versus locomotor training on paired reflex depression of the soleus H-reflex after motor incomplete spinal cord injury. Arch Phys Med Rehabil. 2009;90(7):1218-28.

42. Fouad K, Krajacic A, Tetzlaff W. Spinal cord and plasticity: opportunities and challenges. Brain Res Bull. 2011;2010(84): 337-42.

43. Hodgson JA, Roy RR, de Leon R, Dobkin B, Edgerton VR. Can the mammalian lumbar spinal cord learn a motor task? Med Sci Sports Exerc. 1994;26(12):1491-7.

44. Dolbow DR, Gorgey AS, Moore JR, Gater DR. A report of practicability of a six month home based functional electrical stimulation cycling program for an individual with tetraplegia. J Spinal Cord Med. 2012;35(3):182-6.

45. Mahoney ET, Bickel CS, Elder C, Black C, Slade JM, Apple D Jr, Dudley GA. Changes in skeletal muscle size and glucose tolerance with electricity stimulated resistance training in subjects with chronic spinal cord injury. Arch Phys Med Rehabil. 2005;86(7):1502-4.
46. Gorgey AS, Shepherd C. Skeletal muscle hypertrophy and decreased intramuscular fat after unilateral resistance training in spinal cord injury: case report. J Spinal Cord Med. 2010;33(1):90-5.

47. Gorgey AS, Dolbow DR, Cifu DX, Gater DR. Neuromuscular electrical stimulation attenuates thigh skeletal muscles atrophy but not trunk muscles after spinal cord injury. J Electromyogr Kinesiol. 2013;23(4):977-84.

48. Dolbow DR, Gorgey AS, Cifu DX, Moore JR, Gater DR. Feasibility of home-based functional electrical stimulation cycling: case report. Spinal Cord. 2012;50(2):170-1.

49. Mohr T, Andersen JL, Biering-Sorensen F, et al. Long-term adaptation to electrically induced cycle training in severe spinal cord injured individuals. Spinal Cord. 1997;35(1):1-16.

50. Crameri RM, Weston A, Climstein M, Davis GM, Sutton JR. Effects of electrical stimulation-induced leg training on skeletal muscle adaptability in spinal cord injury. Scand J Med Sci Sports. 2002;12(5):316-22.

51. Skold C, Lonn L, Harms-Ringdahl K, et al. Effects of functional electrical stimulation training for 6 months on body composition and spasticity in motor complete tetraplegic spinal-injured individuals. J Rehabil Med. 2002;34(1):25-32.

52. Fornusek C, Davis GM, Russold MF. Pilot study of the effect of low-cadence functional electrical stimulation cycling after spinal cord injury on thigh girth and strength. Arch Phys Med Rehabil. 2013;94(5):990-3.

53. Chen SC, Lai CH, Chan WP, et al. Increases in bone mineral density after functional electric stimulation cycling exercises in spinal cord injured patients. Disabil Rehabil. 2005;27(22):1337-41.

54. Shields RK, Dudley-Javoroski S. Musculoskeletal plasticity after acute spinal cord injury: effects of long-term neuromuscular electrical stimulation training. J Neurophysiol. 2006;95(4):2380-90.

55. Shields RK, Dudley-Javoroski S. Musculoskeletal adaptations in chronic spinal cord injury: effects of long-term soleus electrical stimulation training. Neurorehabil Neural Repair. 2007;21(2): 169-79.

56. Myers J, Lee M, Kiratli J. Cardiovascular disease in spinal cord injury: an overview of prevalence, risk, evaluation, and management. Am J Phys Med Rehabil. 2007;86(2):142-52. Review.

57. Groah SL, Weitzenkamp D, Sett P, et al. The relationship between neurological level of injury and symptomatic cardiovascular disease risk in the aging spinal injured. Spinal Cord. 2001;39(6):310-7.

58. Bauman WA, Raza M, Chayes Z, et al. Tomographic thallium201 myocardial perfusion imaging after intravenous dipyridamole in asymptomatic subjects with quadriplegia. Arch Phys Med Rehabil. 1993;74(7):740-4.

59. Bauman WA, Spungen AM. Disorders of carbohydrate and lipid metabolism in veterans with paraplegia or quadriplegia: a model of premature aging. Metabolism. 1994;43(6):749-56.

60. Hooker SP, Scremin AM, Mutton DL, Kunkel CF, Cagle G. Peak and submaximal physiologic responses following electrical stimulation leg cycle ergometer training. J Rehabil Res Dev. 1995;32(4):361-6.

61. Perret C, Berry H, Hunt KJ, Donaldson N, Kakebeeke TH. Feasibility of functional electrical stimulated cycling in subjects with spinal cord injury: an energetic assessment. J Rehabil Med. 2010;42(9):873-5.

62. Furlan JC, Fehlings MG. Cardiovascular complications after spinal cord injury: pathophysiology, diagnosis, and management. Neurosurg Focus. 2008;25(5):E131. 